\title{
Persistent Eosinophilia: A Diagnostic Dilemma
}

\author{
Hafsa Shabeer*, Chethana Mannem, Gayathri Bilagali Ramdas and Thejasvi Krishnamurthy \\ Department of Pathology, Kempegowda Institute of Medical Sciences, Bangalore - 560004, Karnataka, India.
}

\section{ABSTRACT}

Chronic eosinophilic leukaemia-not otherwise specified (CEL-NOS) is a myeloproliferative neoplasm associated with an autonomous, clonal proliferation of eosinophilic precursors resulting in persistent eosinophilia.

A 50-year-old female presented with easy fatiguability, cough and generalised swelling of the body. Investigations revealed anaemia with leucocytosis (56.150 x 103/ul) and 88\% eosinophils (absolute eosinophil count was 49412/ul). Peripheral smear showed abnormal eosinophils exhibiting abnormal granulation and nuclear lobation. Reactive causes were ruled out and a bone marrow aspiration/biopsy revealed mildly hypercellular marrow with increased number of eosinophils and their precursors, $7 \%$ blasts along with dysplastic megakaryocytes hypolobated and occasional segmented forms. Molecular studies including chromosomal and gene analysis were done. A combination of the clinical picture, laboratory and molecular studies led us to a diagnosis of CEL-NOS.

The causes for eosinophilia are myriad and range from reactive causes like parasitic infestations to neoplasms in which eosinophils are a part of the neoplastic population/ are cytokine-mediated reactive component in the background of another neoplasm.

The incidence of CEL-NOS is obscure due to significant overlap with Idiopathic Hypereosinophilic Syndrome (IHES). While CEL-NOS is a myeloproliferative neoplasm and its diagnosis can be made provided evidence of a clonality is present, IHES is a diagnosis of exclusion. It is important to differentiate the two entities as they carry different prognosis and modes of treatment.

\section{Keywords: Eosinophilia, Chronic Eosinophilic Leukaemia, Idiopathic Hypereosinophilic Syndrome.}

\section{Introduction}

Chronic eosinophilic leukemia is a myeloproliferative neoplasm characterized by clonal expansion of eosinophilic precursors resulting in persistently elevated numbers of eosinophils in the bone marrow, peripheral blood as well as tissues. ${ }^{1,2}$ The diagnosis of CEL depends on criteria that relies heavily on cytogenetics and molecular studies put forward in the WHO fascicle ${ }^{1}$ after ruling out causes of secondary eosinophilia. In the absence of such evidence, the diagnosis of IHES may be made, which is a diagnosis of exclusion. ${ }^{1,2}$

\section{Case Report}

A 50-year-old female presented with easy fatiguability, cough and generalised swelling of the body of 15 days duration. She had history of intermittent skin rashes over sun exposed areas, and two episodes of loss of consciousness following hot water bath.

General examination showed pallor with bilateral pitting edema of upper and lower limbs. There was generalised thickening of the skin which was particularly prominent over the lower back. Features of dermographism was also present.
Routine blood investigations showed haemoglobin of $8.9 \mathrm{~g} /$ $\mathrm{dl}$, with a total leucocyte count of $56.150 \times 10^{3} / \mathrm{ul}$ with $88 \%$ eosinophils (the absolute eosinophil count being 49412/ul) and the erythrocyte sedimentation rate (ESR) was raised (106mm in the first hour). The peripheral smear showed absolute eosinophilia with a few abnormal eosinophils exhibiting abnormal granulation and nuclear lobation (Fig 1), blood smears did not reveal any haemoparasites. Urinalysis and stool examination were unremarkable. IgE was found to be raised $(781 \mathrm{IU} / \mathrm{ml})$, however antinuclear antibodies and alpha-1-antitrypsin were within normal limits. Serology for HIV, HBsAg and VDRL were non-reactive. The conditions associated with secondary eosinophilia were ruled out.

Ultrasonography of the abdomen revealed mild splenomegaly, and HRCT of the thorax showed cardiomegaly with a thin rim of pericardial effusion while echocardiogram showed right atrial, right ventricular and IVC dilatation.

Bone marrow aspiration revealed increased number of eosinophils and their precursors along with $7 \%$ blasts (Figs 2\&3) and biopsy (Fig 4) showed mildly hypercellular marrow and abnormal megakaryocyte clustering along 
with dysplastic megakaryocytes (Fig 4) showing hypolobation and occasional segmented forms (pawn ball megakaryocytes).

In view of the aforementioned findings, molecular studies were done. Chromosomal analysis was interpreted as 'normal female karyotype', and FISH was negative for PDGFRA deletion/rearrangement. Gene analysis for BCRABL fusion transcripts, JAK2 (exons 12 and 14), MPL (exon 10) and CALR (exon 9) were found to be negative, as were evidence of monoclonal B-cell or T-cell population.

Thus, a diagnosis of CEL-NOS was made based on clinical, laboratory and molecular studies.

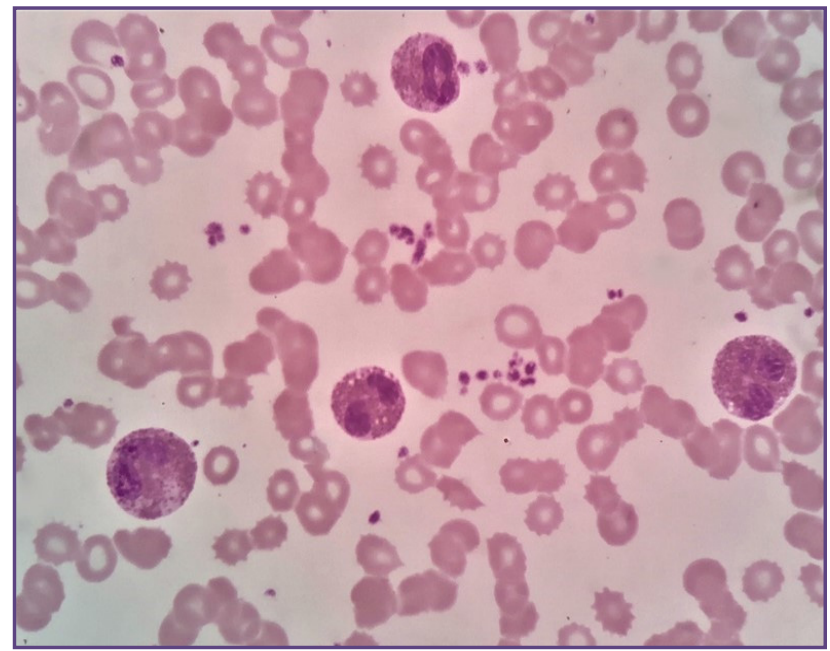

Fig. 1: Peripheral blood eosinophilia exhibiting abnormal patchy granulation and nuclear lobation (Leishman, 1000x).

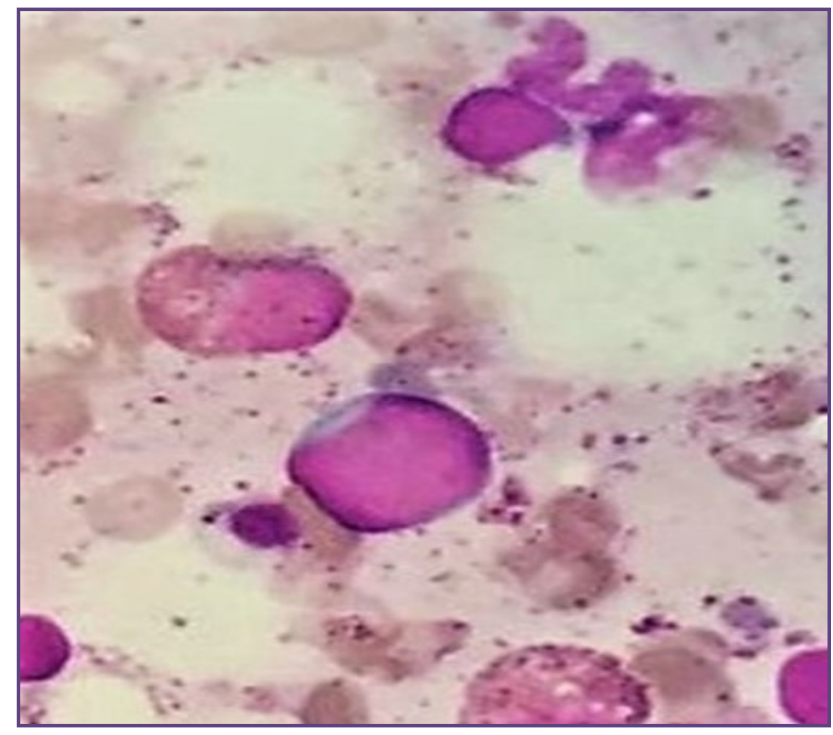

Fig. 3: Bone marrow aspiration showing blasts (MGG, 1000x).

\section{Discussion}

The general consensus for the upper limit of absolute eosinophil count is $0.5 \times 10^{9} / 1 .{ }^{3,4}$ Essentially, the severity of eosinophilia has been divided into mild, moderate and severe with the AEC being 0.5-1.5 x 10\%/1, 1.5-5 x 109/1 and $>5 \times 10^{9} / 1$ respectively. ${ }^{3,4,5,6}$ Abnormal accumulation of eosinophils can have profound and deleterious effects on health by end organ damage due to eosinophil activation and release of various cytokines. ${ }^{4,7}$

Eosinophilia has been broadly classified into familial and acquired. Familial eosinophilia is an autosomal dominant disorder characterised by eosinophilia that may result in

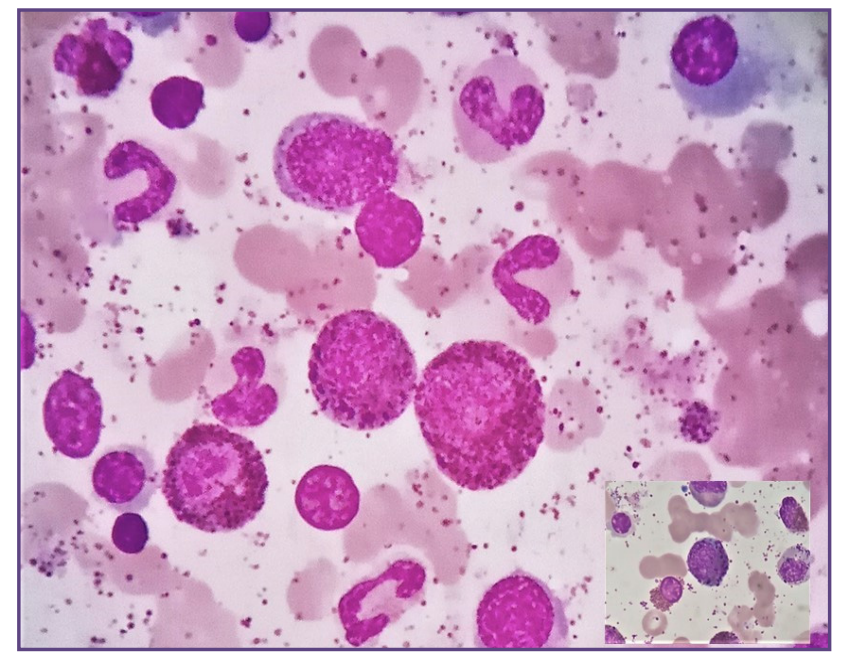

Fig. 2: Bone marrow aspiration showing increased eosinophilic precursors (MGG, 1000x) (Inset: Abnormal eosinophilic precursors, MGG 1000x).

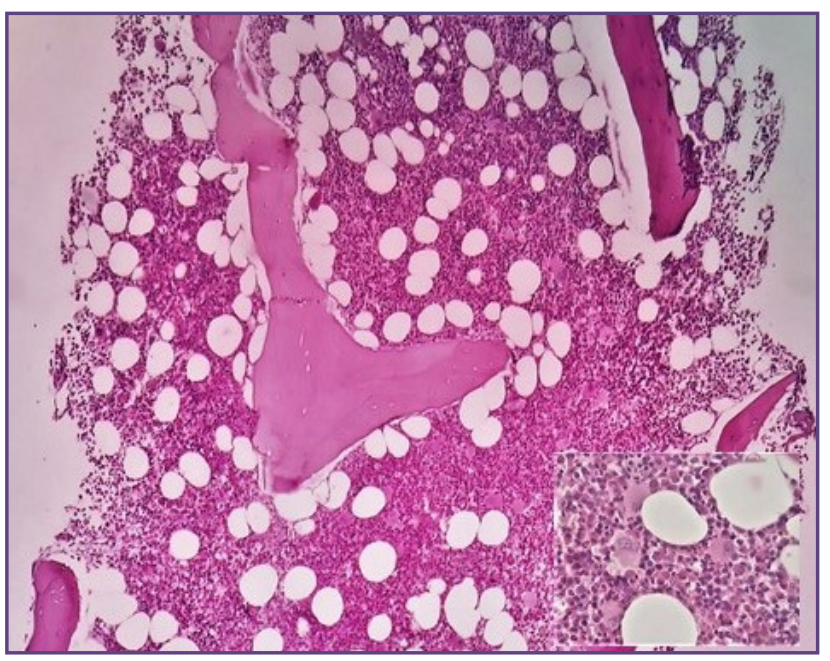

Fig. 4: Bone marrow biopsy showing a mildly hypercellular marrow with clustering of atypical megakaryocytes (H\&E, 40x)[Inset: Cluster of atypical megakaryocytes (H\&E, 400x)]. 
end organ damage in some affected family members. ${ }^{8}$ An underlying genetic defect is rarely responsible for eosinophil activation. ${ }^{8}$ Acquired eosinophilia is classified into secondary (reactive causes), primary (clonal) and idiopathic including hypereosinophilic syndrome. ${ }^{6,9}$

The path to working up patients with hypereosinophilia is arduous, requiring a number of tests to arrive at an accurate diagnosis. A systematic approach with the patients' presenting features serving as a guide would be pragmatic. ${ }^{10}$ A detailed history including drugs, travel history and allergies is mandatory, as many studies implicate intake of herbal medication in eosinophilia. ${ }^{10,11}$ Various parasites such as Strongyloides stercoralis, Ankylostoma duodenale, Schistosoma species etc may cause eosinophilia and must be ruled out. ${ }^{3,10,12} \mathrm{~A}$ diagnosis of CEL can be made if there is evidence of clonality or increased blasts in the peripheral blood/bone marrow, while IHES is a diagnosis of exclusion. ${ }^{13}$

The current WHO guidelines gives weightage to cytogenetic and molecular studies as its primary criteria without a definite outline for morphologic criteria. This becomes problematic especially in cases of CEL without proof of clonality ${ }^{4}$ in whom a false diagnosis of Idiopathic HES may be made according to the current guidelines. ${ }^{13}$

An update to this classification was formulated in 2011 at The Working Conference on Eosinophil Disorders and Syndromes in Vienna, Austria. ${ }^{4}$ Essentially, a multidisciplinary team proposed new definitions and criteria in the hope that it might streamline the current WHO guidelines. More emphasis was given on histomorphology, as many cases tend to fall in the grey zone between CEL and IHES as the diagnosis is based primarily and solely on cytogenetics and molecular studies.

Wang et al ${ }^{14}$ studied 139 patients diagnosed with CEL $(n=17)$ and IHES $(n=122)$, and found that $16 / 17(94 \%)$ cases with CEL and 24/122 (20\%) cases with IHES had abnormal bone marrow morphology. They assessed the bone marrow for cellularity, morphology and numbers of all lineages, myeloid: erythroid ratio and fibrosis, and concluded that the most common aberrations were hypercellularity, abnormal eosinophils, abnormal megakaryocytes, elevated myeloid: erythroid ratio $(>10)$, fibrosis (moderate to marked), dysgranulopoiesis and dyserythropoiesis.

\section{Conclusion}

We present this case report of chronic eosinophilic leukemia as an attempt to highlight the arduous process of working up a patient with hypereosinophilia. A systematic and thorough evaluation, including a detailed history, clinical examination and laboratory investigations will aid in coming to an accurate diagnosis as it is important to differentiate between chronic eosinophilic leukemia and hypereosinophilic syndrome, as they confer different therapeutic and prognostic implications.

\section{Acknowledgements}

We would like to express our gratitude to the late Dr. Adeep for his contribution to working up this case. We would like to thank our technical staff for their expertise, as well as the patient herself for her co-operation.

\section{Funding}

There was no financial support.

\section{Competing Interests}

The authors declare that they have no competing interests.

\section{Reference}

1. Swerdlow S, Campo E, Harris N, Jaffe E, Pileri S, Stein H et al. WHO classification of tumours of haematopoietic and lymphoid tissues. Lyon: International Agency for Research on Cancer; 2017.

2. Bain B, Clark D, Wilkins B. Bone Marrow Pathology. Newark: John Wiley \& Sons, Incorporated; 2019.

3. Tefferi A, Patnaik MM, Pardanani A. Eosinophilia: secondary, clonal and idiopathic. British journal of haematology. 2006 Jun;133(5):468-92.

4. Valent P, Klion AD, Horny HP, Roufosse F, Gotlib J, Weller $\mathrm{PF}$ et al. Contemporary consensus proposal on criteria and classification of eosinophilic disorders and related syndromes. Journal of Allergy and Clinical Immunology. 2012 Sep 1;130(3):607-12.

5. Brigden M, Graydon C. Eosinophilia detected by automated blood cell counting in ambulatory North American outpatients: incidence and clinical significance. Archives of pathology \& laboratory medicine. 1997 Sep 1;121(9):963.

6. Gotlib J, Cools J, Malone JM, Schrier SL, Gilliland DG, Coutré SE. The FIP1L1-PDGFR $\alpha$ fusion tyrosine kinase in hypereosinophilic syndrome and chronic eosinophilic leukemia: implications for diagnosis, classification, and management. Blood. 2004 Apr 15;103(8):2879-91.

7. Gleich GJ. Mechanisms of eosinophil-associated inflammation. Journal of Allergy and Clinical Immunology. 2000 Apr 1;105(4):651-63.

8. Klion AD, Law MA, Riemenschneider W, McMaster ML, Brown MR, Horne M et al. Familial eosinophilia: a benign disorder?. Blood. 2004 Jun 1;103(11):4050-5.

9. Tefferi A. Blood eosinophilia: a new paradigm in disease classification, diagnosis, and treatment. InMayo Clinic Proceedings 2005 Jan 1 (Vol. 80, No. 1, pp. 75-83). Elsevier.

10. Hujoel IA, Jaeger TM. 65-Year-Old Woman With Chronic Eosinophilia. InMayo Clinic Proceedings 2018 May 1 (Vol. 93, No. 5, pp. 646-650). Elsevier. 
11. Tefferi A, Gotlib J, Pardanani A. Hypereosinophilic syndrome and clonal eosinophilia: point-of-care diagnostic algorithm and treatment update. InMayo Clinic Proceedings 2010 Feb 1 (Vol. 85, No. 2, pp. 158-164). Elsevier.

12. Kumar A, Sinha S, Tripathi AK. Chronic eosinophilic leukemia: a case report and review of literature. Indian Journal of Hematology and Blood Transfusion. 2007 Dec $1 ; 23(3-4): 112-5$.
13. Bain BJ. Eosinophilic leukemia and idiopathic hypereosinophilic syndrome are mutually exclusive diagnoses. Blood. 2004 Dec 1;104(12):3836-7.

14. Wang SA, Hasserjian RP, Tam W, Tsai AG, Geyer JT, George TI et al. Bone marrow morphology is a strong discriminator between chronic eosinophilic leukemia, not otherwise specified and reactive idiopathic hypereosinophilic syndrome. haematologica. 2017 Aug 1;102(8):1352-60.

*Corresponding author:

Dr Hafsa Shabeer, \#830, 13th main, 3rd block Koramangala, Bangalore - 560034, Karnataka, India.

Phone: +91809008559876

Email: hafsa.9186@gmail.com

Financial or other Competing Interests: None. 